\title{
Stuttering generalization self-measure: preliminary development of a self- measuring tool
}

Article

Accepted Version

Creative Commons: Attribution-Noncommercial-No Derivative Works 4.0

Alameer, M., Meteyard, L. and Ward, D. (2017) Stuttering generalization self-measure: preliminary development of a selfmeasuring tool. Journal of Fluency Disorders, 53. pp. 41-51. ISSN 0094-730X doi:

https://doi.org/10.1016/j.jfludis.2017.04.001 Available at https://centaur.reading.ac.uk/70184/

It is advisable to refer to the publisher's version if you intend to cite from the work. See Guidance on citing.

To link to this article DOI: http://dx.doi.org/10.1016/j.jfludis.2017.04.001

Publisher: Elsevier

All outputs in CentAUR are protected by Intellectual Property Rights law, including copyright law. Copyright and IPR is retained by the creators or other copyright holders. Terms and conditions for use of this material are defined in the End User Agreement.

www.reading.ac.uk/centaur 
Central Archive at the University of Reading

Reading's research outputs online 


\title{
Stuttering Generalization Self-Measure: Preliminary Development of a Self-Measuring Tool
}

\author{
Mohammad Alameer ${ }^{*}$, Lotte Meteyard ${ }^{2}$, David Ward 2,3 \\ ${ }^{1}$ Fawzia Sultan Rehabilitation Institute, Kuwait \\ 2 University of Reading, United Kingdom \\ 3 Apple House Centre for stammering, United Kingdom
}

Objectives: The reader will become knowledgeable about 1) the relationship between stuttering severity and speech-anxiety level, and 2) the importance of assessing the generalization effect in different social speaking situations. Additionally, the reader will understand the processes of validating the Stuttering Generalization Self-Measure.

\begin{abstract}
Introduction: Generalization of treatment is considered a difficult task for clinicians and people who stutter (PWS), and can constitute a barrier to long-term treatment success. To our knowledge, there are no standardized tests that collect measurement of the behavioral and cognitive aspects alongside the client's self-perception in real-life speaking situations.
\end{abstract}

Purpose: This paper describes the preliminary development of a Stuttering Generalization SelfMeasure (SGSM). The purpose of SGSM is to assess 1) stuttering severity and 2) speech-anxiety level during real-life situations as perceived by PWS. Additionally, this measurement aims to 3) investigate correlations between stuttering severity and speech-anxiety level within the same reallife situation.

Method: The SGSM initially reported includes nine speaking situations designed that are developed to cover a variety of frequent speaking scenario situations. However, two of these were less commonly encountered by participants and subsequently not included in the final analyses. Items were created according to five listener categories (family and close friends, acquaintances, strangers, persons of authority, and giving a short speech to small audience). Forty-three participants (22 PWS, and 21 control) aged 18 to 53 years were asked to complete the assessment in real-life situations.

Results: Analyses indicated that test-retest reliability was high for both groups. Discriminant validity was also achieved as the SGSM scores significantly differed between the controls and PWS two groups for stuttering and speech-anxiety. Convergent validity was confirmed by significant correlations between the SGSM and other speech-related anxiety measures.

Key Words: Stuttering severity, speech-anxiety, self-perception, assessment, generalization. 


\section{Highlights}

- It is important to consider assessing stuttering in naturalistic speaking situations

- The relationship between stuttering and related anxiety can influence the outcome

- SGSM can discriminate between PWS and controls in terms of fluency and speech anxiety levels

- SGSM can serve as desensitizing treatment agent

- SGSM can assess stuttering and related anxiety within the same speaking situation 


\section{Introduction}

Stuttering is associated with both motoric and psychological symptoms. The motoric symptoms may include disrupted airflow while speaking (e.g., Peters, Hietkamp, \& Boves, 1993), disrupted laryngeal function especially while initiating voice (e.g., Logan, 2003; Viswanath \& Rosenfield, 2000), and disrupted oral muscles used to articulate speech sounds (e.g., McClean, Tasko, \& Runyan, 2004). Stuttering is also associated with speech or social anxiety (Menzies, et al., 2008). Some studies suggest that fear from speaking is associated with the listener's reactions and evaluations of the speaker (e.g., Blumgart, Tran, Craig, 2010; Klein \& Hood, 2004). Numerous studies highlight that PWS demonstrate anxiety however this anxiety is generally restricted to social performance-based speaking situations (Craig \& Tran, 2006; Iverach et al, 2011, Menzies et al, 1999; St Clare et al, 2009). Such symptoms can also be found in fluent speakers, but with different rates. According to Wingate (2002) and Van Riper (1982), speakers who do not stutter can present with speech dysfluencies, which may be exacerbated in more stressful speaking situations.

Existing stuttering measures tend to target both the motoric and the psychological components of stuttering. However, these measures collect data pertaining to stuttering in controlled or structured settings (such as in the clinic). Finn (2003) and St Louis (2006) suggested that such procedures may inhibit individuals from exhibiting their real behavior since highly controlled settings abstract them from their natural environments. Hence, it is crucial to observe stuttering and its related behaviors in speaking situations that occur beyond the clinical environment.

Guitar (2014) postulated that standard assessment must occur on both the subjective and objective levels. A subjective assessment includes gathering information, interviewing, and administering some general questionnaires. Here, the clinician can closely observe both speech and attitudes towards stuttering as well as gather qualitative information about the participant's 
environment. Additionally, this initial assessment serves to establish rapport. An objective assessment, on the other hand, takes place to measure both stuttering and any speech-related anxiety. The outcome from these measures is used to set a baseline and then track the progress during the treatment phase and follow up stages. Assessment targets several aspects of speech, such as frequency, type, duration, and severity of stuttering behaviour (e. g., Stuttering Severity Instrument-4, SSI-4, Riley, 2009). Additionally, assessments can target speech naturalness, speech rate, and physical concomitant behaviors (Riley, 2009).

\subsection{Incorporating functional measures with treatment}

The term personally significant has been recently introduced by Bothe and Richardson (2011) to highlight the outcomes that are demonstrably of value to the client undergoing treatment. The authors asserted that it should be up to the client to judge whether or not a treatment can help in reaching desired outcomes. Ingham, Ingham, and Bothe (2012) added that the value of the term centers on the distinction between the clinician's judgment and the client's self-evaluation of the treatment outcome. However, this requires valid and reliable assessment procedures of the treatment outcomes. Kazdin (2011) also raised the issue of clinical significance, and stated:

"The usual way of measuring validity is showing that the scores on a measure correlate with performance elsewhere, but this does not address the matter. . clearly [reflect] a difference that is important in the lives of the clients? How does one know? For some of the measures, such as subjective evaluation, perceiving that there is a difference defines an important change. For other measures, very little assessment work has been completed to show that huge changes on a measure or being closer to a normative sample and further away from a dysfunctional sample has palpably improved the client's everyday functioning” (pp. 319-320).

To date, most stuttering measures do not satisfy Kadzin's (2011) point of view (Ingham et al., 2012). Most existing measures, for both speech and non-speech (attitude) behaviors, are used to reflect treatment outcomes without looking at the connection between the measure itself with treatment from a more functional perspective. Hence, Ingham et al (2012) emphasized three 
important elements that should be included in stuttering assessment: functional self-measures, within- and beyond-clinic speaking tasks, and repeated assessments that occur before, during and after treatment.

\subsubsection{Functional self-measures}

Some studies indicate that PWS can achieve greater fluency after using self-measuring treatment strategies (Finn, 1997; Finn, Howard, \& Kubala, 2005). Hence, self-measurement can be an additional component in treatment that can alter the motoric and psychological aspects in stuttering (Cullinan \& Prather, 1968; Eve, Onslow, Andrews, 1995; Martin \& Haroldson, 1992; Onslow, Andrews, \& Costa, 1990). For the client, such self-evaluation measures can include stuttering severity, speech naturalness and anxiety level, most commonly evaluated via Likert scales. Ingham et al. (2012) added that self-measurement can be more powerful when both the client and clinician select the targets within treatments. Additionally, it is important to mention the power of self-modeling, which involves the recording and subsequent review of successful (problem-free) performance. Bandura (1997) suggested that self-modeling can improve selfbelieve, and this in turn can lead to improved fluency outcomes. For example, video selfmodeling after speech restructuring treatment was linked with improvements in self-reporting outcomes (Cream et al, 2010; Harasym, Langevin, \& Kully, 2015).

\subsection{Functional within-clinic and beyond-clinic tasks}

A number of studies pertaining to stuttering treatments include both within-clinic and beyondclinic measures (e.g., Bothe, Davidow, Bramlett, \& Ingham, 2006; Bothe \& Richardson, 2011; Curlee, 1993; Ingham et al., 2012; James, 1981; Jones et al., 2005; Onslow, Costa, \& Rue, 1990; R. J. Ingham, \& Costello, 1984, 1985; R. J. Ingham, \& Cordes, 1999). However, most of these studies do not provide justification for the selection of the speaking tasks since they assume that 
collecting samples from random within-clinic and beyond-clinic speaking situations genuinely reflects clinical significance. Hence, Bothe and Richardson (2011) argued that in order to achieve a personally significant outcome, the selection of these tasks should come from the client, rather than being purely researcher - or clinician - driven. The combination of self-measuring and selfselection can provide changes in both dysfluency and related attitudes. Moreover, a number of researchers (e.g., Ingham, \& Costello, 1984, 1985; Curlee, 1993; \& Ingham, \& Cordes, 1999) emphasized the importance of beyond-clinic measurements, as PWS can be more fluent in-clinic, but not beyond-clinic. Other studies found reduced stuttering when treatment techniques were used in beyond-clinical speaking situations (e.g., James, 1981, Jones et al., 2005; Onslow, Costa, \& Rue, 1990).

\subsubsection{Functional repeated measuring}

Repeated assessments (before, during, and after treatment) are an important component for stuttering treatment (Ingham \& Riley, 1994). For PWS, pretreatment assessment can provide a baseline, from which the client can realize the behaviors that need to be modified, and then visualize the changes through ongoing assessments. Hence, such continuous assessment procedures can enhance treatment effects (Ingham et al, 2012).

\subsection{Overview of stuttering assessments}

People who stutter generally report decreased fluency in real-life communication in comparison with clinical sessions (Finn, 2003), which is why standardized assessment is a vital element in all treatment plans to track the progress of PWS both within and beyond clinical settings (American Speech-Language and Hearing Association, 1995; Hillis, 1993; Yaruss, 2001). St. Louis (2006) suggested that stuttering measurements should include four components: 1) affective (targeting generalization in real-life); 2) behavioral (targeting speech manner and 
fluency level); 3) cognitive (targeting the emotions related to stuttering); and 4) self-perceptual (targeting the individual perception of his own stuttering). To our knowledge, there are no standardized assessments that provide objective measurements of both stuttering severity and speech-anxiety whilst engaging in real-life speaking circumstances. In other words, most of the measures target stuttering severity and speech anxiety levels from within clinical settings or through self-reports PWS. The lack of functional measures may reflect difficulties associated with observing their natural speaking environments. Additionally, stuttering behaviors can vary significantly across situations, time, and listeners, making the process of obtaining a valid measurement complicated (Bloodstein, 1995; Manning, 2001).

Currently, there are a variety of assessment tools that measure both speech and speech anxiety in PWS. The Unhelpful Thoughts and Beliefs About Stuttering (UTBAS) was developed by St Clare, et al. (2009) to assess unhelpful thoughts and beliefs about stuttering that are related to social anxiety. This tool is a valid and reliable measure in discriminating the social anxiety level of PWS from those who do not, and it is considered to be sensitive in assessing responses in PWS pre- and post- CBT treatment. It includes 66 self-report items, comprised of 27 items that make specific reference to stuttering (e.g., 'People will doubt my ability because I stutter'), and 39 items that make no reference to stuttering (e.g., 'I am incompetent'). However, it lacks the assessment of speech anxiety during real-life situations, as it is primarily designed to measure the negative thoughts about stuttering in general, rather than while engaging in real or specific situations.

Riley (2009) developed the Stuttering Severity Instrument-4 (SSI-4) for clinical and research purposes, which has been established as a reliable tool to measure the frequency and duration of dysfluencies, physical concomitants, and the overall severity of stuttering during the clinical session. Similarly, this instrument only assesses the fluency of PWS within clinical settings, with only one component (phone conversation) to be assessed beyond-clinic. 
There are other tools that assess the communication attitudes of PWS in specific social situations. The Inventory of Interpersonal Situations (IIS) (Van Dam-Baggen \& Kraaimaat, 1999) (e.g., Asking a friend to help you with something), the Self Perceived Communication Competence Scale (SPCC) (McCroskey \& McCroskey, 1988) (e.g., talk in a large meeting of friends), the Willingness to Communicate Scale (WTC) (McCroskey \& Richmond, 1987) (e.g., talk with a friend while standing in line), and the Personal Report of Communication Apprehension (PRCA) (McCroskey, 1982) (e.g., 'ordinarily I am very tense and nervous in conversations'), are all measurements that assess social anxiety in interpersonal situations. Participants are asked to rate their anxiety level based on their general experience about specific social situations. Although these tools target specific social situations, the assessment of the participants' stuttering and anxiety during performing real social situations is overlooked.

\subsection{The development of a Stuttering Generalization Self-Measure (SGSM)}

The purpose of this paper is to describe the preliminary development of the SGSM (see Appendix A). Since current stuttering measuring tools have overlooked the aspect of assessing fluency and anxiety associated with stuttering in real and beyond-clinic situations, the purpose of developing the SGSM is to 1) assess stuttering severity and 2) assess speech-anxiety levels during real-life situations as perceived by PWS. Additionally, this measurement aims to 3) investigate correlations of stuttering severity and speech-anxiety level within the same real-life situation such as speaking to a family member, to an acquaintance, and to a stranger in various conversational settings.

The items of the SGSM were created to fulfill specific criteria in testing functional social situations. As Bloodstein (1995) and Manning (2001) suggested, stuttering severity can vary according to the nature of situation and the conversation partner. Therefore, the items of SGSM were developed to cover a wide array of frequently occurring social situations, with a variety of 
conversation partners or listeners. We began with situations identified in existing aassessmnts. The SPCC (McCroskey \& McCroskey, 1988), which assesses self-perceived competence in four contexts (dyadic, meeting, group, and public) with respect to three targets (stranger, acquiescence, and friend). The OASES (Yaruss \& Quesal, 2008) asks individuals to rate the difficulty of speaking across a wide range of situations (e.g. small groups, large groups, telephone conversations, talking with co-workers, telling stories or jokes). The development of the Self Efficacy Scaling by Adult Stutterers (ESAS, Ornstein \& Manning, 1985) identified common day to day speaking situations (e.g. introducing friends, ordering food) and then reduced situations to make sure there was no redundancy.

As the SGSM is a self-report measure, we needed to include situations that were likely to occur frequently in day-to-day life. In order to make sure that the measure was completed, we also needed to keep the number of items relatively low, and the method of self-report straightforward. Participants would be required to complete the measure without the presence of a clinician. Whilst this introduces a lack of control, it attempts to introduce ecological validity into the measuring speech ability and anxiety away from the clinic. Finally, to ensure construct validity the items for the SGSM were constructed to allow comparison against two other measures (the SPCC and WTA).

To meet these needs, we created nine generic items to cover different contexts (e.g., phone conversation, face-to-face conversation, groups) and different audiences (e.g., family and close friends, acquaintances, strangers, people of authority; see Appendix 1). Within the SGSM, items 1,2 , and 3 represent the category of close friends and family, items 4 and 5 represent the category of acquaintances, items 6 and 7 represent the category of strangers, item 8 represents the category of person of authority, and item 9 represent the category of public speech. The final two items were made optional: speaking with people of authority (item 8) and giving speech to an audience (item 9). This was because they are two circumstances that are unlikely to occur very frequently 
to be considered as daily speaking situations. These items could be considered if they represent in the participant's daily life, where frequent formal meetings and public speaking take place (e.g. teaching or work presentations).

For content validity, items from SGSM were compared to items from the WTC (McCroskey \& McCroskey, 1988) SPCC (McCroskey \& McCroskey, 1988) and IIS (Van DamBaggen \& Kraaaimaat, 1999). Face validity was established as the listener categories of the SGSM (family/close friend, acquaintance, stranger, group, and phone call) match those of the mentioned tools (WTC, SPCC, IIS). We were unable to assess inter-item reliability as the SGSM contains only one item for each situation. That is, each item assesses the fluency and anxiety level when engaging in a social situation with different level of difficulty.

When using the self-assessment measure, Participants are asked to rate their anxiety before the event and then retrospectively rate their anxiety during the event. Retrospective report of affective states is used frequently in other questionnaires (e.g. providing ratings for "today", "past few days" or "the past week") (Watson, Clark \& Tellegen, 1988) and in clinically administered assessments that evaluate how individuals feel about stuttering and the impact it has on them (e.g. Yaruss \& Quesal, 2008). Providing ratings close to the event (before and after) will confound these ratings with one-another, therefore they are combined in the final score to give an overall measure of anxiety.

\section{Methodology}

\subsection{Participants}

PWS and control participants were recruited through social media and word-of-mouth. Additionally, two speech therapy clinics (Fawzia Sultan Rehabilitation Institute, and Salem Alali Speech and Hearing Center) were contacted to include some of their waiting-list clients. To standardize the SGSM, normative data included 43 participants, where $46.5 \%$ were females ( $n=$ 
$20)$ and $53.5 \%$ were males $(n=23)$. Participants aged in range from 18 to 53 years $(M=26.19$, $M d n=25, S D=8.4)$. Participants were distributed into two groups. The PWS group included 22 PWS $(n=22$, males $=14$, females $=8)$. The age range in this group was 18 to $35(M=23.7$, $M d n=23, S D=5.09)$. The control group included 21 control participants $(n=21$, males $=9$, females $=12)$ who ranged in age from 18 to 53 years $(M=28.8, M d n=26, S D=10.4)$.

The investigators explained what stuttering is (e. g., types) to ensure accuracy and consistency in identification of stuttering moments as opposed to normal speech dysfluencies. Participants in the control group were helped to identify normal dyfluency is (e. g., hesitations, fillers, revisions, loss of control, etc.), and that people who do not stutter can demonstrate these daily, with different levels depending on the type of conversation/listerner. The investigators demonstrated a sample of stuttering and normal dysfluencies to participants to make sure they are identified and evaluated properly prior to performing the SGSM beyond-clinic. Moreover, clinics explained how to rate the self-perception of related anxiety, and how different speaking situations and different listeners can create different levels of speech-anxiety (e. g., speaking to a family member versus speaking to a person of authority).

The inclusion criteria for this study included participants who: 1) were at least eighteen years old; 2) had not received speech andlor anxiety treatment; 3) had no physical, neurological, or cognitive deficits; 4) were Arabic speakers; and 5) lived in Kuwait, where the study was conducted.

\subsection{SGSM administration procedures}

In the current study, participants were required to engage in the first seven speaking situations. Items 8 and 9 (speaking with a person of authority, and giving a short presentation) were eliminated for the current study, as they did not occur sufficiently frequently on a daily basis. There was no specific order to complete the speaking situations, as items were to be filled 
as they occurred in each participant's daily life. To avoid the influence of items on each other, Participants were encouraged to spread completing the speaking situations through the entire administration period (10 days) and not finishing them in one day (e.g., 1 item per day). Participants were asked to complete and return the evaluation package in seven to ten days. Before engaging in the situation, participants were asked to rate their anxiety level using a fivepoint Likert scale ( $1=$ no anxiety and $5=$ severe anxiety) that was related to that specific situation (e.g., phone conversation with a stranger). Participants were then asked to record the conversation with their smart-phone or digital sound recorder. The recording was asked to last for at least 30 seconds of their continuous talking for each situation. During the conversation, the participant was asked to note their anxiety level (e. g., write it down, or keep it in mind). Ideally, the conversation partner should have been notified of the study, and a consent form should be signed. However, notifying the conversation partner would hinder the naturalness of the conversation, and it would prolong the administration period. Therefore, to avoid this ethical issue (i.e., preserve the conversation partner's anonymity), recordings were deleted as soon as the participant's performance was scored.

After completing each situation, participants were asked to record the level of anxiety during the speaking situation using the same Likert scale ( $1=$ no anxiety and $5=$ severe anxiety). A total anxiety score was created by adding the two Likert scales together (maximum anxiety score of 10 per situation, total score of 70 across the 7 speaking situations). Participants were also asked to listen to the recording after completing each situation, rating their stuttering severity using a nine-point likert scale $(1=$ no stuttering and $9=$ severe stuttering $)$. In the comment section, participants were asked to provide a brief description about each situation (e.g., for the conversation with a group of family members, participants may have described it as a conversation with their mother and sister about a summer vacation). Finally, participants were 
asked to hand in or send their SGSM form and speech-recordings to the clinician as soon as all items had been completed (up to ten days).

Perceived stuttering could influence the rating of anxiety level during the conversation or speaking situation, making it more likely that these would be correlated. To provide a more objective measure of stuttering, the main investigator also calculated a Stuttering Severity Percentage (SS\%). After receiving both the form and recordings, the main investigator listened to the conversations to assign a Stuttering Severity Percentage (SS\%) for each situation, where the total of stuttered syllables is divided by the total number of syllables within the speech sample. Scores from the SGSM allowed for comparisons of a more objective dysfluency count with the participants' self-rating of their own stuttering. Additionally, these scores allowed for possible correlations between stuttering and anxiety associated with each specific social situation.

\subsection{Validity}

A larger sample size would have generated greater statistical power, however, as Jones, Val Gebski, Onslow, and Packman (2002) noted, recruiting a large number of participants who fit these inclusion criteria is often difficult owing to the relatively low prevalence of communication disorders, such as stuttering. Therefore, in order to achieve convergent validity, participants completed the SGSM in conjunction with the WTC (McCroskey \& McCroskey, 1988), SPCC (McCroskey \& McCroskey, 1988), and IIS (Van Dam-Baggen \& Kraaaimaat, 1999). The four measurements were given to each participant in a booklet form. To avoid order effects, the sequence of presenting the four tests within the booklet was randomized.

To achieve discriminant validity, scores from the stuttering group were compared with those of the control group. This allowed for the discrimination between speech and anxiety behaviors related to speaking social situations between the two groups. 


\subsection{Reliability}

For intra-judge reliability, a sample of the SS\% for both PWS $(n=10)$ and control participants $(n=10)$ was blindly calculated twice. The sample was selected by taking odd participant numbers. For inter-judge reliability, the same sub-sample was blindly recalculated by another qualified speech-language therapist.

Test-retest reliability was used to assess the stability of SGSM over time. The SGSM was re-administered a month after the initial testing. Ideally, a power analysis should have taken place to determine the sample size needed for retesting. However, as the number of participant was relatively small, all participants were retested on the SGSM.

\subsection{Data Analysis}

Discriminant validity was measured by using the Mann-Whitney test to assess whether there was a significant difference between the two groups. Convergent validity was measured by using a series of Spearman's rho tests to assess the correlation between SGSM and the other measures (IIS,Van Dam-Baggen \& Kraaaimaat, 1999; SPCC, McCroskey \& McCroskey, 1988; and WTC, McCroskey \& McCroskey, 1988) in measuring speech anxiety. Spearman's rho was also used to explore the relationship (strength and direction) between stuttering and speech anxiety within SGSM for specific speaking situation (non-parametric variables). Spearman's rho was used as it was assumed that the two variables (stuttering and speech anxiety) are measured on an interval and ordinal scales. Additionally, it was assumed that stuttering and speech anxiety create a monotonic relationship (increased stuttering is associated with increased speech anxiety).

As SS\% is continuous data, for both intra and inter judge reliability we calculated the interclass correlation coefficient (ICC) across participants. For inter-judge reliability, we also calculated the concordance correlation coefficient (Lin, 1989). For test-retest reliability, we followed Rousson, Gasser \& Seifert (2002) and calculated the correlation (Spearman's Rho) for 
each participant for each measure (SS\%, Fluency, Anxiety before and Anxiety during), averaged across all situations in the SGSM.

\section{Results}

\subsection{Observer Reliability for \%SS}

For intra-rater reliability, results indicated a strong level of agreement between the raters, as the ICC was $0.95(95 \% \mathrm{CI}=0.92-0.98, n=20)$. Similarly, inter-rater reliability, the ICC was $0.95(95 \% \mathrm{CI}=0.92-0.98, \mathrm{n}=20)$ and the concordance correlation coefficient was $0.998(n=20$, $95 \%$ confidence interval $=0.9995-0.9998)$.

\subsection{Validity analyses}

Discriminant validity was achieved as the SGSM scores significantly differed between the two groups. The stuttering group had, on average, higher SS\% $(M=9.5, S D=4.14$, range $=3.73-$ $18.75)$ and speech anxiety scores $(M=41, S D=11.72$, range $=22-58)$ than the control group (SS\%: $M=0.25, S D=1.06$, range $=0-4.9$; Total speech-anxiety: $M=22.6, S D=6.7$, range $=14$ 34). These differences were significant (SS\%: Mann-Whitney $U=36.5, p<0.001$; Total speech anxiety: Mann-Whitney $U=41, p<0.001)$.

For the entire sample, correlations between SGSM and the other measures are included in Table 1. Scores on the SGSM were strongly correlated with scores on SPCC (McCroskey \& McCroskey, 1988) and WTC (McCroskey \& McCroskey, 1988) (SPCC, $r=0.793, p<0.001$; WTC, $r=0.830, p<0.001)$. These findings supported the convergent validity of the scale. However, correlation between SGSM and IIS (Van Dam-Baggen \& Kraaaimaat, 1999) was not significant (IIS, $r=0.130, p=0.405$ ), and scores on the IIS were only moderately correlated with the WTC $(r=0.398, p=0.008)$ and the SPCC (McCroskey \& McCroskey, 1988) $(r=0.389, p=$ $0.010)$. 
Table 1

Bivariate Correlations of SGSM with IIS, SPCC and WTC, $n=43$

\begin{tabular}{lc}
\hline & SGSM \\
\hline IIS & .13 \\
SPCC & $.79^{* *}$ \\
WTC & $.83^{* *}$ \\
\hline
\end{tabular}

$* p<.05$ (2-tailed)

$* * p<.01$ (2-tailed)

3.3. Relationship between stuttering (independently assessed) and speech anxiety in the SGSM

Spearman's correlation showed that stuttering (SS\%) and overall perceived speech anxiety were not correlated for the PWS group $(r=0.296, p=0.182)$. Fig. 1 . demonstrates the correlation between stuttering and speech-anxiety for PWS group.

Figure 1

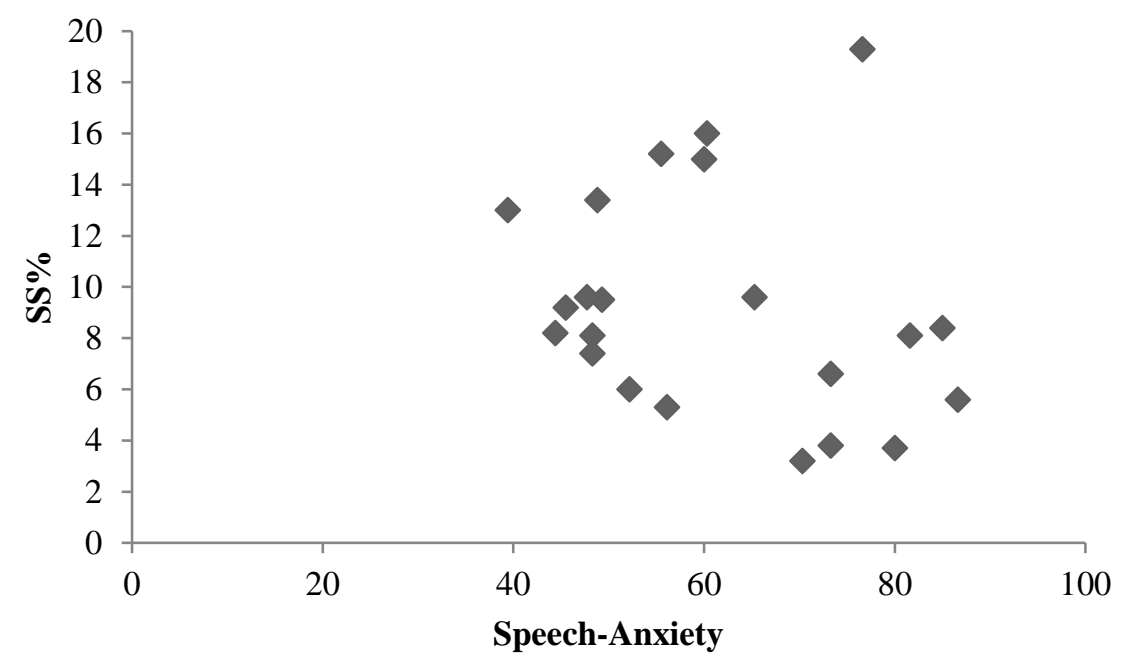

Fig. 1. The relationship between stuttering and speech anxiety in PWS group, $n=22$ 


\subsubsection{SGSM self-ratings of anxiety: speaking with strangers}

For PWS, neither perceived stuttering $(r=0.29, p=0.194)$ nor SS $\%(r=0.06, p=0.804)$ were significantly correlated with overall speech anxiety reported on the SGSM.

Table 2

Bivariate correlations among SGSM sub-sections when speaking with strangers

\begin{tabular}{lll}
$\begin{array}{l}\text { Correlation } \\
\text { coefficient }\end{array}$ & SS\% & $\begin{array}{l}\text { Perceived } \\
\text { stuttering }\end{array}$ \\
\hline $\begin{array}{l}\text { Total speech } \\
\text { anxiety for whole } \\
\text { sample }\end{array}$ & $.69 * *$ & $.72 * *$ \\
$\begin{array}{l}\text { Total speech } \\
\text { anxiety for PWS }\end{array}$ & .06 & .29 \\
\hline
\end{tabular}

$* p<.05$ (2-tailed)

$* * p<.01$ (2-tailed)

\subsubsection{Speaking with acquaintances}

For the PWS group, overall speech anxiety was not strongly associated with $\mathrm{SS} \%(r=0.21, p=$ $0.360)$ or perceived stuttering $(r=0.35, p=0.110)$.

Table 3

Bivariate correlations among SGSM sub-sections when speaking with acquaintances

\begin{tabular}{llc}
$\begin{array}{l}\text { Correlation } \\
\text { coefficient }\end{array}$ & SS\% & $\begin{array}{l}\text { Percei } \\
\text { stutter }\end{array}$ \\
\hline $\begin{array}{l}\text { Total speech } \\
\text { anxiety for whole } \\
\text { sample }\end{array}$ & $.70^{* *}$ & $.69^{* *}$ \\
$\begin{array}{l}\text { Total speech } \\
\text { anxiety for PWS }\end{array}$ & .21 & .35 \\
\hline
\end{tabular}

${ }^{*} p<.05$ (2-tailed)

$* * p<.01$ (2-tailed) 


\subsubsection{Speaking with family/close friends}

For the PWS group in this SGSM sub-section, SS\% $(r=0.48, p=0.025)$ and perceived stuttering ( $r=0.44, p=0.039)$ were significantly related to overall anxiety.

Table 4

Bivariate correlations among SGSM sub-sections when speaking with family/close friends

\begin{tabular}{lll}
$\begin{array}{l}\text { Correlation } \\
\text { coefficient }\end{array}$ & $\mathrm{SS} \%$ & $\begin{array}{l}\text { Perceived } \\
\text { stuttering }\end{array}$ \\
\hline
\end{tabular}

\begin{tabular}{lll}
$\begin{array}{l}\text { Total speech } \\
\text { anxiety for whole }\end{array}$ & $.69^{* *}$ & $.68^{* *}$ \\
sample & & \\
$\begin{array}{l}\text { Total speech } \\
\text { anxiety for PWS }\end{array}$ & $.48^{*}$ & $.44^{*}$ \\
\hline
\end{tabular}

$*_{p}<.05$ (2-tailed)

$* * p<.01(2$-tailed $)$

\subsection{Test-retest reliability}

All participants were retested on the SGSM approximately one month after initial testing. Since the entire sample was tested, no power analysis was performed. The correlation coefficient (Spearman's Rho) for SS\% between first and second testing was 0.99, for Fluency 0.98, for Anxiety before 0.98 and for Anxiety during 0.97 .

\section{Discussion}

Stuttering measurement tools should try to incorporate four main components (St. Louis, 2006): 1) generalization in real-life; 2) speech manner and fluency level; 3) emotions and beliefs that are related to stuttering; and 4) the individual's perception of his/her own stuttering. In the current study, the SGSM was developed to measure stuttering and anxiety in real-life through a self-assessment and self-report process. This novel measuring tool aims to assess both stuttering 
and speech-anxiety within real-life speaking situations, in addition to providing the opportunity for clients to rate their own fluency within each specific task.

Results indicate that the SGSM can discriminate between stuttering and non-stuttering individuals in terms of speech dysfluency and speech-anxiety levels, showing a significant difference between the two groups on both measures. Those who stuttered had, on average, more severe dysfluencies and speech anxiety during speaking situations relative to non-stuttering individuals. These findings are consistent with previous research (e.g., Iverech et al., 2011; St. Clare et al., 2007). Despite concerns that asking individuals to report anxiety and perceived stuttering would confound these two measures, correlational analyses showed no significant correlations between measures of stuttering (SS\% and perceived stuttering) and reported anxiety for speaking with strangers and acquaintances (for PWS and Controls). However, there was a significant correlation between measure of stuttering (SS\% and perceived stuttering) and reported anxiety for the PWS group when speaking with friends and close family. We can tentatively infer that individuals are likely to experience more anxiety with strangers and acquaintances (and report this as such), but the level of stuttering may be relatively stable. In contrast, individuals are likely to experience less anxiety with friends and close family and these lower reported levels of anxiety may then genuinely correlate with rates of stuttering. This suggests two things. First, the self-reported assessment of stuttering is relatively reliable as it always patterned with the externally judged SS\%. Second, the SGSM appears to be a useful tool in developing selfmodeling strategies in therapy (Bandura, 1997). Individuals can be asked to compare their expected and experiences anxiety with their actual performance in stuttering.

The SGSM demonstrated convergent validity with similar measures such as SPCC (McCroskey \& McCroskey, 1988) and WTC (McCroskey \& McCroskey, 1988) for the entire sample. These two measures have similar categorizations of speaking situations to the ones in the SGSM (speaking to family member(s)/ close friend(s), acquaintance(s), stranger(s), and having a 
phone conversation); these categories were all strongly positively correlated with the ones of SGSM for both groups. However, The SGSM did not correlate with IIS (Van Dam-Baggen \& Kraaaimaat, 1999). A possible explanation for this is that the SGSM targets specific speaking situations that are categorized into three categories, while the IIS measure social-anxiety in general. Moreover, scores on the WTC and SPCC were not correlated with those on IIS as strongly as they did with the SGSM. These findings support the speculation about the differences between IIS and the other measures used in this study. Therefore, it appears that the SGSM can measure speech anxiety similarly to other valid and reliable tests, in addition to providing a novel contribution to measuring stuttering and the anxiety associated with stuttering in PWS. In other words, other speech-anxiety measures such as SPCC and WTC assess speech-anxiety, as they are perceived by the examinee, while SGSM provides a real-time assessment during a specific set of speaking situations, which are categorized into different levels of difficulty. Additionally, the SGSM can measure the relationship between stuttering and speech-anxiety in each specific speaking situation.

One of the main objectives of developing the SGSM was to measure the relationship between stuttering and speech-anxiety in specific social situations. Although results indicated a strong relationship between the two measures overall, the relationships were not significant when each group was assessed separately. Therefore, future research would do well to target a larger sample for an extended period of time to further explore the relationship between these two variables.

For the PWS group, the test-retest reliability of the instrument was not significantly different for SS\% but was significantly different for speech-anxiety in the PWS group, as participants presented with less speech-anxiety in the second testing occasion. This difference between the two testing periods in total speech anxiety could be explained by the fluctuations of speech-anxiety levels in PWS that resulted from speaking with different listeners in the first 
testing occasion than the ones in the second testing occasion. It is also possible that having a different level of speech-anxiety in the second test was the result of familiarity with the items of the test and the familiarity of self-rating their own anxiety. Regardless, total speech anxiety levels were similar at the two testing periods. On the other hand, the control group's scores were not significantly different at the two testing occasions. As predicted, the control group scored more consistently across the different speaking situations. However, we found a marginal difference in perceived speech anxiety at test-retest time points (1 month apart) for the Controls. The group means were similar so this was driven by a few controls reporting higher anxiety. Further exploration of the test-retest reliability of the SGSM is needed.

To our knowledge, the SGSM is the first tool measuring stuttering and speech-anxiety levels simultaneously with real-life speaking situations. Although this study only presents preliminary findings, the outcomes of the SGSM seem promising. As Finn (2003) suggested, the generalization and transition between clinical settings and the real world could be a large step for PWS. Therefore, given that the SGSM captures stuttering severity and speech anxiety beyond the clinical setting, it could be a useful connection between these two stages in that the SGSM can be used to track a client's progress after receiving any given treatments that target stuttering and speech anxiety. Additionally, this tool could be used to compare the progress of therapy at any given The SGSM can also be used in conjunction with other measuring tools such as the UTBAS (Iverech et al., 2011) for speech-anxiety and the SSI-4 (Riley, 2009) for stuttering. This is useful for researchers and clinicians who wish to collect both objective and subjective information about clients in both within and beyond clinical settings.

\subsection{Implications}

A number of researchers in the field of speech dysfluencies advocate for the assessment of stuttering and related speech anxiety within and beyond clinical settings (e.g., Bothe et al, 2006; 
Bothe \& Richardson, 2011; Kadzin, 2011; St Louis, 2006). This originated from frequent complaints from clients who generally felt that treatment effects were more obvious with their clinicians than they were in everyday speaking situations - i.e., it is not uncommon for clients to speak relatively fluently with their clinicians, but then stutter and face severe anxiety outside the clinic. While clinicians may believe that their clients have met their treatment goals given their more fluent speech within the clinic, clients themselves may not be satisfied given their increased dysfluency in their everyday lives. Therefore, measuring fluency and speech-anxiety in nonclinical settings is crucial in determining the direction and progress of any given treatment.

The SGSM was developed to cover a variety of speaking situations with different types of listeners to address previous findings that stuttering can vary in severity depending on the social context (Bothe \& Richardson, 2011; Ingham, 2012). The participants in this study, on average, exhibited less stuttering and speech anxiety while speaking with family members or close friends, and increased stuttering and anxiety while performing in social situations with strangers. For this reason, assessing the client in-clinic only can be misleading.

Another addition of the SGSM is that it can be a beneficial agent for treatment. As suggested by numerous researchers (Finn, 1997; Finn, Howard, \& Kubala, 2005; Ingham, 2012), PWS were able to reach perceptually more fluent speech after using self-measuring treatment strategies. Selfmeasuring tools likely allow clients to be more mindful of their strengths and weakness, which allows them to set more realistic treatment targets.

\subsection{Limitations and directions for future research}

The SGSM was developed to assess stuttering frequency and self-perceived stuttering with its related anxiety cohesively in beyond-clinic speaking situations. This tool can provide an original contribution to the field of stuttering assessment. Although the introduction of this tool is believed to be of significance, its development is still in its preliminary stages, therefore some 
improvements are needed. First, it is important that the type and duration of dysfluencies are discussed within the collected speech sample. Numerous researchers indicate that the type of dysfluency can determine the severity of the disorder, and can distinguish PWS from fluent speakers (e.g., Susca \& Healy; Yairi \& Ambrose, 2013). For example, within a speech sample, longer stutters, or blocks, are considered more severe than short pauses. It is also important to consider other factors that can influence the severity of the disorder, such as revisions. The sample should also include a measurement of the duration of dysfluencies, as they can provide important information about the degree to which stuttering is interfering with daily communication. For example, blocks are more severe than shorter stutters; these need to be addressed within studies (Myers, 1987; Preus, 1981; Riley, 1994, 2009; Van Riper, 1982).

Additionally, the relatively small sample size likely affected the results of this study, particularly when assessing the relationship between stuttering and speech-anxiety. Therefore, future research would do well to recruit a larger sample. Third, more items could be included to cover a wider range of speaking situations. This addition will potentially serve in increasing interitem reliability (e. g., speaking with a salesman in a store or placing an order at a restaurant).

Some participants reported that the administration time for the SGSM (approximately 7 days) was not enough. Ideally, the SGSM can be administered within 2-3 days, but it seemed difficult for some participants, especially in the PWS group to complete it in less than a week. Participants expressed concerns about the difficulty in performing the tasks of SGSM, especially when they spoke to strangers. Although the speaking situations in the SGSM were developed to match the ones in daily life, some participants in the stuttering group were not comfortable completing the test. It is believed that these participants were heavily anxious about performing, knowing that the conversations were to be recorded and analyzed later. It is important to point out that such difficulty is expected from PWS; therefore, performing this task can be a part of the therapeutic process. Interestingly, participants were able to complete the SGSM on the second 
occasion with relatively more ease, as they were able to complete it in less than a week with minimal feedback from the clinician.

Another issue that could have affected the validity of this test was the possible bias in choosing the recordings by participants. It is possible that participants may have chosen recordings where they have less dysfluencies to return to clinicians. Similarly, bias in selecting the speaking situations was possible, as participants may have chosen to record (e. g., speaking with a friend that was angry, versus, speaking with a friend that was relaxed). Hence, it is important to consider controlling the choice recordings and speaking situations by participants.

\section{Conclusion}

The SGSM is, to our knowledge, the first tool designed to measure stuttering and speechanxiety simultaneously within real-life speaking situations. Preliminary findings suggest that high stuttering severity is associated with high levels of speech anxiety. This study also indicates that the SGSM can discriminate between PWS and their fluent peers in terms of both fluency and speech-anxiety levels since PWS demonstrated significantly higher levels of both dysfluency and speech-anxiety. Additionally, the SGSM demonstrates that the type of speaking situation can impact the degrees of stuttering and speech anxiety in PWS. 


\section{Appendix A}

Stuttering Generalization Self-Measure

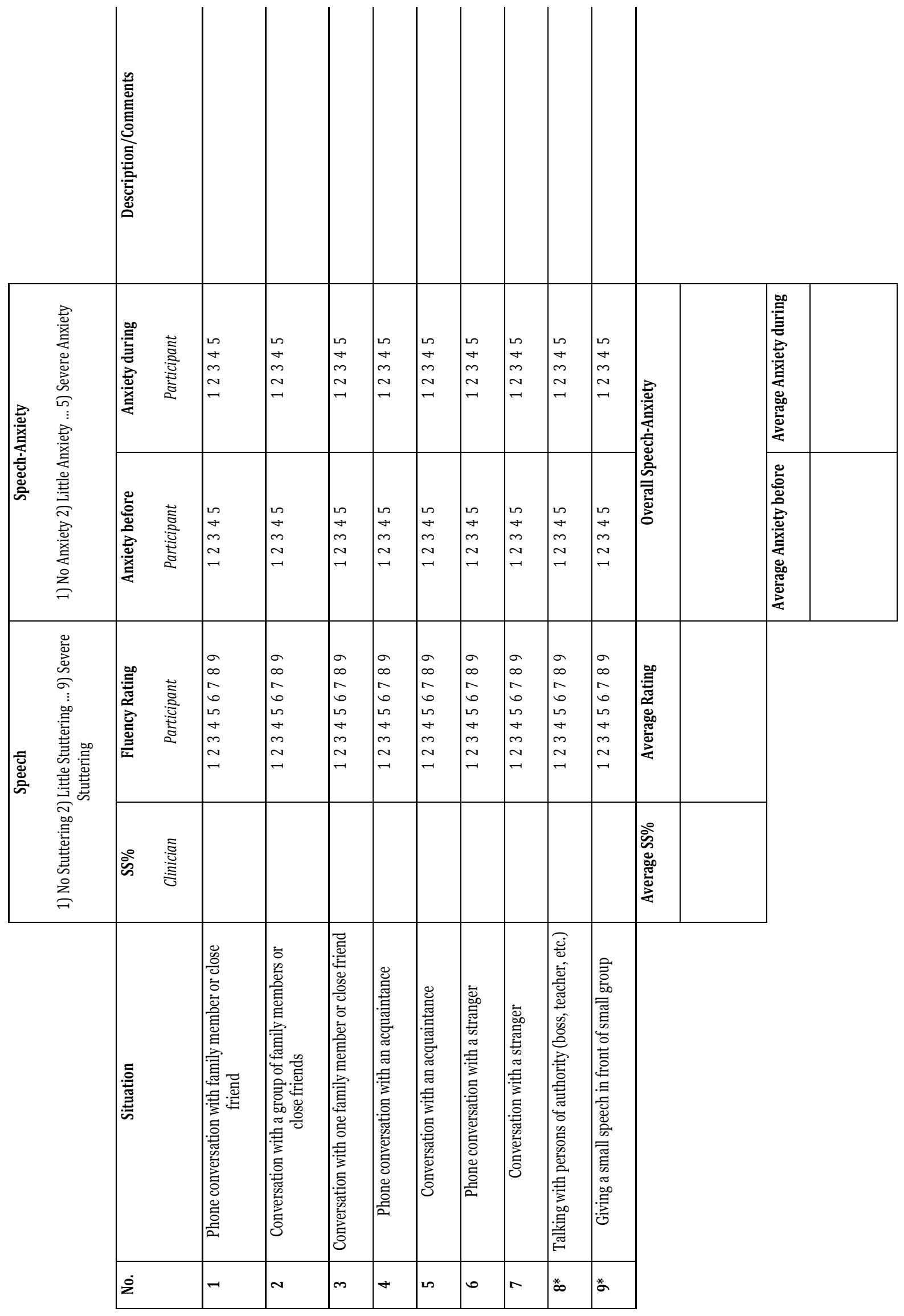




\section{References}

American Speech-Language-Hearing Association (1995). Guidlines for practice in stuttering treatment. American Speech-Language-Hearing Association, 37(suppl. 14), 26-35.

Bandura, A. (1997). Self-efficacy: The exercise of control. New York: Freeman.

Bloodstein, O. (1995). A Handbook on Stuttering (5th ed.). San Diego, CA: Cingular Publishing Group, Inc.

Blumgart, E., Tran, Y., \&, Craig, A (2010). Social anxiety disorder in adults who stutter. Depression and Anxiety, 27, 687-692.

Bothe, A. K., \& Richardson, J. D. (2011). Statistical, practical, clinical, and personal significance in speech-language pathology. American Journal of Speech-Language Pathology, 20, 233-242.

Bothe, A. K., Davidow, J. H., Bramlett, R. E., Franic, D. M., \& Ingham, R. J. (2006). Stuttering treatment research, 1970-2005: II. Systematic review incorporating trial quality assessment of pharmacological approaches. American Journal of SpeechLanguage Pathology, 15, 342-352.

Cullinan, W. L., \& Prather, E. M. (1968). Reliability of "live" ratings of the speech of stutterers. Perceptual \& Motor Skills, 27, 403-409.

Curlee, R. F., (1993). Evaluating treatment efficacy for adults: Assessment of stuttering disability. Journal of Fluency Disorders, 18, 319-331

Craig, A., \& Tran, Y. (2006). Fear of Speaking: Chronic anxiety and stuttering. Advances in Psychiatric Treatment, 12, 6.

Cream, A., O’Brian, S., Jones, M., Block, S., Harrison, E., Lincoln, M., et al. (2010). Randomized controlled trial of video self-modeling following speech restructuring treatment of stuttering. Journal of Speech, Language, and Hearing Research, 53, 887-897. 
Eve, C., Onslow, M., Andrews, C., \& Adams, R. (1995). Clinical measurement of early stuttering severity: The reliability of a 10-point scale. Australian Journal of Human Communication Disorders, 23, 26-39.

Finn, P. (1997). Adults recovered from stuttering without formal treatment: Perceptual assessment of speech normalcy. Journal of Speech, Language, and Hearing Research, 40, 821-831.

Finn, P. (2003). Addressing generalization and maintenance of stuttering treatment in schools: A critical look. Journal of Communication Disorders, 36, 153-164.

Finn, P., Howard, R., \& Kubala, R. (2005). Unassisted recovery from stuttering: Selfperceptions of current speech behavior, attitudes, and feelings. Journal of Fluency Disorders, 30, 281-305.

Guitar, B. (2014). Stuttering: An integrated approach to its nature and treatment (4rd Ed.). Baltimore, PA: Lippincott Williams \& Wilkins.

Harasym, J., Langevin, M., \& Kully, D. (2015). Video self-modeling as a post-treatment fluency recovery strategy for adults. Journal of Fluency Disorders, 44, 32-45.

Hillis, J. (1993). On-going assessment in the management of stuttering: A critical perspective. Journal of Speech Language Pathology, 2(1), 24-37.

Ingham, J. C., \& Riley, G. (1998). Guidelines for documentation of treatment efficacy for young children who stutter. Journal of Speech, Language, and Hearing Research, $41,753-770$.

Ingham, R., Ingham, J. C., Bothe, A. K. (2012). Integrating functional measures with treatment: A tactic enhancing personality significant change in the treatment of adults and adolescents who stutter. American Journal of Speech-Language, Pathology, 21, 264-277. 
Ingham, R. J., \& Cordes, A. K. (1999). On watching a discipline shoot itself in the foot: Some observation on current trends in stuttering treatment. In N. B. Ratner \& E. C. Healey (Eds.), Stuttering research and practice: Bridging the gap (pp. 211-230). Mahwah, NJ: Erlbaum.

Ingham, R. J., \& Costello, J. M. (1984). Stuttering treatment outcome evaluation. In J. M. Castello (Ed.), Speech disorders in children: Recent advances (pp. 313-346). San Diego, CA: College-Hill Press.

Ingham, R. J., \& Costello, J. M. (1985). Stuttering treatment outcome evaluation. In J. M. Castello (Ed.), Speech disorders in children: Recent advances (pp. 189-223). San Diego, CA: College-Hill Press.

Iverach, L., Menzies, R. G., O'Brian, S., Packman, A., Onslow, M. (2011). Anxiety and stuttering: Continuing to explore a complex relationship. American Journal of Speech-Language Pathology, 20, 231-232.

James (1981). Behavioral self-control of stuttering using time-out from speaking. Journal of Applied Behavioral Analysis, 14, 25-37

Jones, M., Gebski, V., Onslow, M., and Packman, A. (2002). Statistical power in stuttering research: A tutorial. Journal of Speech, Language, \& Hearing Research, 45, 243255.

Jones, M., Onslow, M., Packman, A., Williams, S., Ormond, T., Schwartz, I., et al. (2005). Randomised controlled trial of Lidcombe Programme of early stuttering intervention [Electronic version]. British Medical Journal, 331(7518), 659.

Kazdin, A. E. (2011). Single-case research designs (2nd ed.). Oxford, England: Oxford University Press.

Klein, J. F., \& Hood, S. B. (2004). The impact of stuttering on employment opportunities and job performance. Journal of Fluency Disorders, 29, 255-273. 
Lin, L. (1989). A concordance correlation coefficient to evaluate reproducibility. Biometrics. International Biometric Society, 45(1): 255-268. doi:10.2307/2532051.

Logan, K. (2003). The effect of syntactic structure upon speech initiation times of stuttering and nonstuttering speakers. Journal of Fluency Disorders, 28, 17-35.

Manning, W. H. (2001). Clinical decision making in fluency disorders (2nd Ed.). Calgary, Canada: Singular Thomson Learning.

Martin, R., \& Haroldson, S. (1992). Stuttering and speech naturalness: Audio and audiovisual judgments. Journal of Speech \& Hearing Research, 35, 521-528.

McClean, M. D., \& Runyan, C. M. (2000). Variations in the relative speeds of orofacial structures with stuttering severity. Journal of Speech, Language, \& Hearing Research, 43(6), 1524-1531.

McCroskey, J. C. (1982). An introduction to rhetorical communication (4th ed.). Englewood Cliffs, NJ: Prentice-hall.

McCroskey, J. C., and McCroskey, L. L. (1988). Self-report as an approach to measuring communication competence. Communication Research Reports, 5, 108-113.

McCroskey, J. C., Richmond, V. P. (1987). Willingness to communicate. In J. C. McCroskey \& J. A. Daly (Eds). Personality and interpersonal communication, 119, 131.

Menzies, R. G., Onslow, M., \& Packman, A. (1999). Anxiety and stuttering: Exploring a complex relationship. American Journal of Speech-Language Pathology, 8, 3-10.

Onslow, M., Andrews, C., \& Costa, L. (1990). Parental severity scaling of early stuttered speech: Four case studies. Australian Journal of Human Communication Disorders, $18,47-61$.

Onslow, M., Costa, L., \& Rue, S. (1990). Direct early intervention with stuttering: Some preliminary data. Journal of Speech and Hearing Disorders, 55, 405-416 
Ornstein, A.F., \& Manning, W. H. (1985). Self-efficacy scaling by adult stutterers. Journal of Communication Disorders, 18(4), 313-320

Peters, H. F., M., Hietkamp, R. K., \& Boves, L. (1993). Aerodynamic and phonatory processes in dysfluent speech utterances of stutterers. American Speech \& Hearing Association, 35, 144.

Riley, G. (1994). Stuttering severity instrument for children and adults. Austin, TX: Pro-ed.

Riley, G. (2009). Stuttering Severity Instrument for Children and Adults- (4th Ed.). Austin, Texas: Pro-ed.

Rousson, V., Gasser, T., \& Seifert, B. (2002). Assessing intrarater, interrater, and test-retest reliability of continuous measurements. Statistics in Medicine, 21(22), 3431-3446

St. Clare, T., Menzies, R. G., Onslow, M., Packman, A., Thompson, R., and Block, S. (2009). Unhelpful thoughts and beliefs linked to social anxiety in stuttering: Development of a measure. International Journal of Language and Communication Disorders, 44 (3), 338-351.

St Louis, K. (2006). Measurement issues in fluency disorders. In N. B. Ratner \& J. Tetnowski (Eds.), Current issues in stuttering research and practice (pp. 61-86). Mahwah, NJ: Erlbaum.

Susca, M, \& Healey, E. (2002). Listener perception along a fluency - disfluency continuum: A phenomenological analysis. Journal of Fluency Disorders, 27, 135-161.

Van-Dam Baggen, R., and Kraaimaat, F. (1999). Assessing Social Anxiety: The Inventory of Interpersonal Situations (IIS). European Journal of Psychology Assessment, 15(1), 25-38.

Van Riper, C. (1982). The nature of stuttering (2nd Ed.). Englewood Cliffs, Nj: Prentice Hall. 
Viswanath, N. S., \& Rosenfield, D. B. (2000). Preponderance of lead voice onset time in stutterers under varying constraints. Communication Disorders Quarterly, 22, 4955.

Watson, D., Clark, L. A., \& Tellegen, A. (1988). Development and validation of of brief measures of positive and negative affect: The PANAS Scales. Journal of Personality and Social Psychology, 54(6), 1063-1070

Wingate, M. (2002). The nature of stuttering (2nd Ed.). San Diego, CA: Academic Press.

Yairi, E., \& Embrose, N. (2013). Epidemiology of stuttering: $21^{\text {st }}$ Century advances. Journal of Fluency Disorders, 38, 66-87

Yaruss, S. (2001). Evaluation treatment outcomes for adults who stutter. Journal of Communication Disorders, 34, 90-115.

Yaruss, S., \& Quesal, R. W., (2008). OASES: Overall Assessment of the Speaker's Experience of Stuttering: Adult. Bloomington, MN: Person Assessment 